\title{
Emerging Clues of Regulatory Roles of Circular RNAs through Modulating Oxidative Stress: Focus on Neurological and Vascular Diseases
}

\author{
Lingfei Li $\mathbb{D},{ }^{1}$ Zhumei Ni $\mathbb{D}^{2},{ }^{2}$ Xiaoli Si $\mathbb{D},{ }^{3}$ Lin Jiang $\mathbb{D},{ }^{1}$ Hongfei Sang $\mathbb{D},{ }^{1}$ Wenqing Xia $\mathbb{D},{ }^{1}$

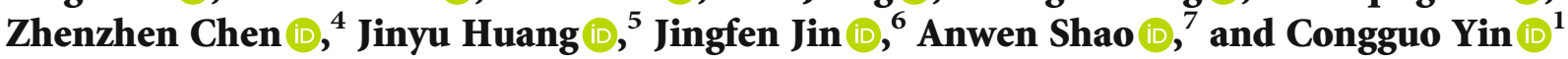 \\ ${ }^{1}$ Department of Neurology, Affiliated Hangzhou First People's Hospital, Zhejiang University School of Medicine, Hangzhou, China \\ ${ }^{2}$ The Fourth Clinical Medical College, Zhejiang Chinese Medicine University, Hangzhou, China \\ ${ }^{3}$ Department of Neurology, The Second Affiliated Hospital, School of Medicine, Zhejiang University, Hangzhou, China \\ ${ }^{4}$ Department of Hematology, Affiliated Hangzhou First People's Hospital, Zhejiang University School of Medicine, Hangzhou, China \\ ${ }^{5}$ Department of Cardiology, Affiliated Hangzhou First People's Hospital, Zhejiang University School of Medicine, Hangzhou, China \\ ${ }^{6}$ Department of Nursing, The Second Affiliated Hospital, College of Medicine, Zhejiang University, Hangzhou, Zhejiang, China \\ ${ }^{7}$ Department of Neurosurgery, The Second Affiliated Hospital, School of Medicine, Zhejiang University, Hangzhou, China
}

Correspondence should be addressed to Anwen Shao; 21118116@zju.edu.cn and Congguo Yin; yincg716@aliyun.com

Received 8 October 2020; Revised 6 February 2021; Accepted 18 February 2021; Published 2 March 2021

Academic Editor: Hidenori Suzuki

Copyright $\odot 2021$ Lingfei Li et al. This is an open access article distributed under the Creative Commons Attribution License, which permits unrestricted use, distribution, and reproduction in any medium, provided the original work is properly cited.

\begin{abstract}
Circular RNAs (circRNAs) are novel noncoding RNAs that play regulatory roles in gene expression. Dysregulation of circRNAs is associated with the development and progression of several diseases, such as diabetes mellitus, nervous system diseases, cardiovascular diseases, and cancer. CircRNAs functionally participate in cell physiological activities through various molecular mechanisms. However, these molecular mechanisms are unclear. Oxidative stress is an essential factor in the pathogenesis of various diseases, including neurological diseases. Emerging roles of circRNAs have been identified in different systems in response to oxidative stress. In this review, we summarize the current understanding of circRNA biogenesis, properties, expression profiles, and the clues indicating the regulatory roles of circRNAs through oxidative stress in various systems, especially the nervous system.
\end{abstract}

\section{Introduction}

Circular RNAs (circRNAs) are a novel type of noncoding RNAs [1]. Instead of having a $5^{\prime}$ cap and $3^{\prime}$ tail structure like linear RNAs, circRNAs have a covalent loop structure, which is stable and evolutionally conserved $[2,3]$. They are diverse and widespread throughout different eukaryotic cells [3]. One of the prominent roles of circRNAs is to regulate gene expression, and thus, they modulate progression in various diseases such as diabetes, nervous system diseases, cardiovascular diseases, and cancer $[4,5]$. Molecular mechanisms of gene expression regulation by circRNAs include microRNA (miRNA) sponge effect, posttranscriptional regulation, and translation regulation $[6,7]$. Notably, the progression of neu- rovascular diseases, one of the most prevalent and devastating diseases affecting adults worldwide [8], is linked to circRNAs widely associated with various pathological responses, including atherosclerosis, neuroinflammation, apoptosis, and neurogenesis [9-11]. Moreover, these circRNAs can be diagnostic, monitoring, therapeutic, and/or prognostic tools in the management of several diseases [12]. Oxidative stress refers to the pathological process of excessive production of reactive oxygen species (ROS) and/or weakened antioxidant capacity of the organism, resulting in an imbalance between ROS production and clearance and, consequently, accumulation of ROS in the body and oxidative damage of cells. This pathological process is closely related to neurovascular diseases. Various risk factors of 
neurovascular diseases, such as smoking [13], fluctuation of blood glucose levels [14], hypertension [15], and hyperhomocysteinemia [16], can all increase ROS production. Accumulating evidence indicates that circRNAs are involved in the process of oxidative stress [17] and may be an important mechanism linking oxidative stressinducing pathological factors to associated pathological conditions of neurological diseases. Exploration of circRNAs and their correlated effects in neurovascular diseases may facilitate accurate diagnosis and serve as new therapeutic targets for the disease. Therefore, we reviewed the emerging clues of the regulatory role of circRNAs through oxidative stress in neurovascular systems.

\section{CircRNAs: Discovery, Features, Biogenesis, and Classification}

The presence of circRNAs in the cytoplasm of eukaryotic cells was observed under an electron microscope in the 1970s [18, 19], and later, circular transcripts of the testisdetermining gene Sry were found in adult mice [20]. However, at that time, circRNAs were only considered products of missplicing, so their functions were underestimated [21]. With the help of high-throughput sequencing technology, a large number of circRNAs have been discovered and characterized [3]. The expression profile of circRNAs presents with three characteristics: (i) abundance: circRNAs are widely found in different cell types and organisms, such as Caenorhabditis elegans, Drosophila, mice and monkeys [22-24], plants [25, 26], and even protists [27-29]; (ii) stability: without $5^{\prime}-3^{\prime}$ polarity and the covalent closed-loop structure of polyadenylate, circRNAs have high resistance to RNA exonuclease or ribonuclease $\mathrm{R}[30,31]$. Moreover, circRNA isomer transcripts have a half-life of more than $48 \mathrm{~h}$; therefore, they are more stable than the related linear transcripts with a half-life of less than $20 \mathrm{~h}$ [3]; and (iii) conservation: circRNAs are evolutionarily conserved among humans, mice $[11,32,33]$, and other mammals $[34,35]$ and even in insects $[29,32,34]$. Nowadays, it is realized that circRNAs are purposefully synthesized and generated by backsplicing, which is covalently linking free $3^{\prime}$ and $5^{\prime}$ ends $[2,36-40]$. Based on the different proportion of exons and introns in the structure, circRNAs can be classified into three categories: exonic circRNAs (ecircRNAs) [3, 6, 32, 41], circular intronic RNAs (ciRNAs) [41], and exon-intron circRNAs (EIciRNAs) [42]. Five hypothesized models of circRNA biogenesis are illustrated in Figure 1: (i) lariat-driven circularization model: during heterogeneous nuclear RNA transcription, the RNA partially folds close to the nonadjacent exon, which leads to an exon skipping event, which results in covalent splices from the $3^{\prime}$ end of a splice donor to the $5^{\prime}$ end of a splice acceptor, forming an exon-containing lariat structure. Afterwards, the introns are removed, thus forming an ecircRNA (Figure 1(a)) [3]; (ii) intron pairing-driven circularization model (also known as "direct backsplicing") [39]: alternative splicing to a circular molecule is presumably based on the pairing of complementary motifs in the transcripts, which may bring two exons closer to each other, resulting in an ecircRNA or
EIciRNA after removing or retaining certain introns (Figure 1(b)) [3, 5]; (iii) ciRNA formation model: this is a novel model that occurs due to failure in debranching, which depends on a 7-nucleotide (nt) guanine uracil-rich element near the $5^{\prime}$ splice site and an 11-nt cytosine-rich element near the branch point (Figure 1(c)) [37, 41]; (iv) RNA binding protein- (RBP-) dependent cyclization model: by the interaction between RBPs, flanking introns in RNA molecules can be bridged causing the formation of circRNAs. Muscleblind (MBL) proteins and quaking proteins are two typical RBPs to mediate the circularization (Figure 1(d)); and (v) variable cyclization model: a single gene locus could produce multiple circularized exons. Competition of RNA pairing within individual introns or across flanking introns can alter exon circularization, which is evolutionarily dynamic (Figure 1(e)) [43].

\section{Molecular Mechanisms of circRNAs in Gene Expression Regulation}

3.1. CircRNAs Act as a Sponge for miRNAs or Competitive Endogenous RNAs (ceRNAs). Several miRNA response elements are involved in mediating circRNAs competitively binding to miRNAs. CircRNAs are thus defined as miRNA sponges as they can "absorb" (i.e., binding to) miRNAs (Figure 2(a)) [7]. They regulate gene expression through binding to miRNAs and inhibit miRNA binding to messenger RNA (mRNA) in the cytoplasm [7, 44]. A pioneer study by Hansen et al. demonstrated that the circRNA ciRS-7 has a highly enriched miRNA binding site for miR-7 [7], which is a microRNA involved in numerous pathways and diseases $[45,46]$. They also discovered that circRNAs from the mouse Sry gene have 16 putative target sites for miR-138 and work as sponges for this miRNA [7]. Recently, increasing evidence demonstrated that circRNAs work as sponges to regulate gene expression in neurovascular diseases. CircGRM4 acts as a sponge and is significantly increased in the eyes of cystathionine $\beta$-synthase-deficient mice. This results in the excessive turnover of the metabotropic glutamate receptor 4 in response to extremely high levels of circulating glutamate which mediates neurovascular toxicity [46]. However, a recent study showed that most circRNAs may not function as miRNA sponges [47]. Therefore, it is possible that the interactions between circRNAs and miRNAs are not simply inhibition-related but related to storage, sorting, and localization of miRNAs [48].

3.2. CircRNAs Interact with RBPs and mRNAs. In addition to the interaction with miRNAs, circRNAs also have a mutual effect on RBPs, including binding, storing, or sequestering RBPs to subcellular locations and acting as competing elements (Figure 2(b)). In 2013, circMBL was demonstrated to bind to MBL resulting in competition with canonical linear splicing and regulation of genes [49]. CircRNA poly(A) binding protein nuclear 1 (circPABPN1) can bind to human antigen $\mathrm{R}(\mathrm{HuR})$ and prevent HuR binding to PABPN1 mRNA, thus affecting translation. This is the first example of competition between a circRNA and its cognate mRNA for an RBP that affects translation [50]. In the same year, Yang et al. 


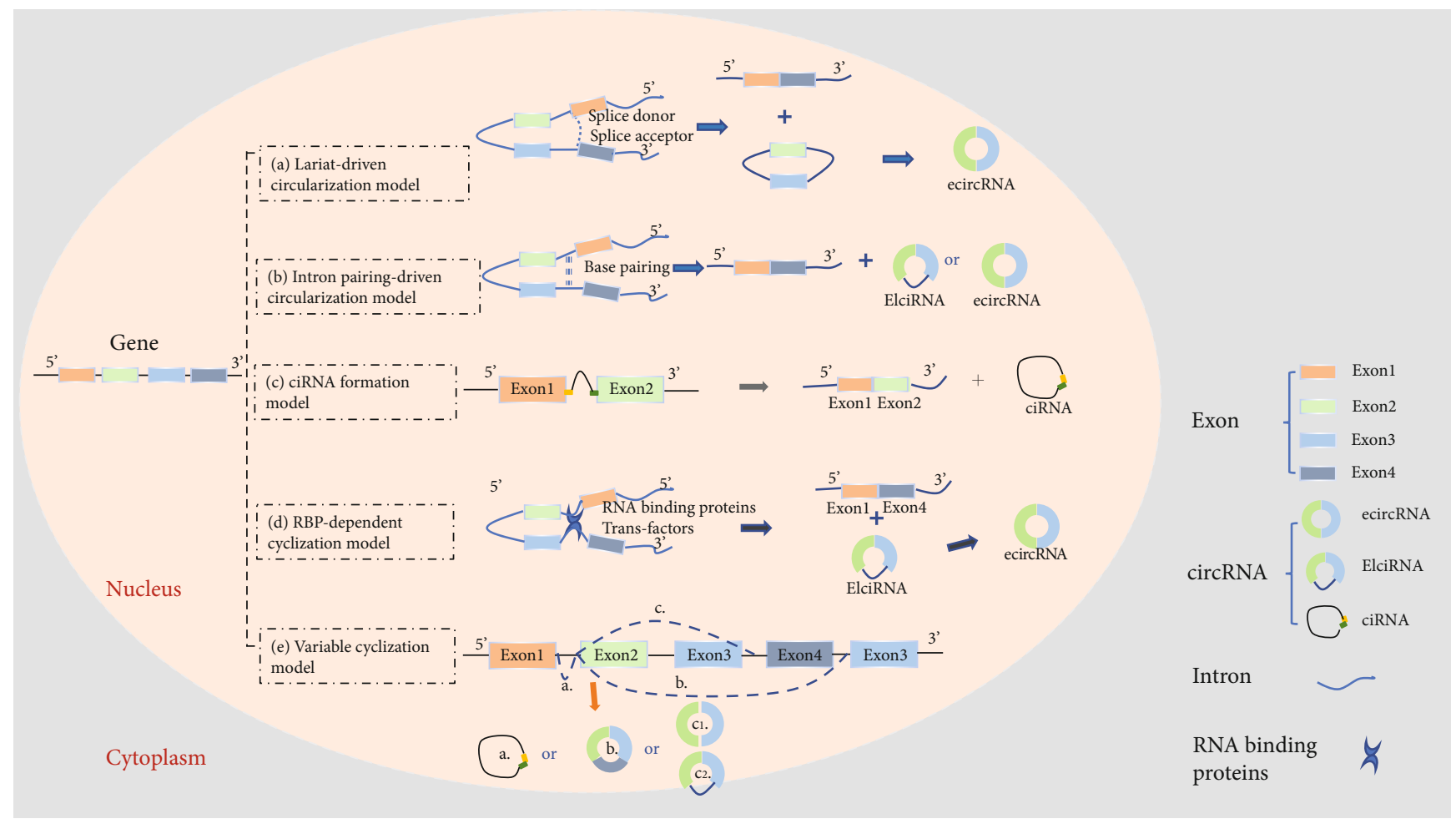

FiguRE 1: Schematic diagram illustrating five models of circRNA biogenesis. (a) Lariat-driven circularization model: the $3^{\prime}$ splice donor of exon 1 and the $5^{\prime}$ splice acceptor of exon 4 link up end-to-end by exon skipping and form an exon-containing lariat structure. Finally, the ecircRNA forms after introns are removed. (b) Intron pairing-driven circularization model: direct base pairing of introns forms a circulation structure, thereby forming ecircRNA or EIciRNA after intron removal. (c) ciRNA formation model: the elements near the splice site escape debranching stably so that the intron lariat is formed from the splicing reaction. (d) RNA binding protein- (RBP-) dependent cyclization model: RBPs bridge two flanking introns close together and then remove introns to form circRNAs. (e) Variable cyclization model: splicing selection and exon circularization may be influenced by inverted repeated Alu pairs (IRAlus) and the competition between them.

found that circAmotl1 can enhance nuclear translocation of the signal transducer and activator of transcription 3 to promote its interaction with the DNA (cytosine-5)-methyltransferase 3A (Dnmt3a) promoter and facilitate the transcription of Dnmt3a [51]. Oxidative stress may also be regulated by circRNAs through interactions with RBPs. circRNA forkhead box O3 (circFoxo3) could bind to cell cycle-associated proteins, cyclin-dependent kinase 2 and $\mathrm{p} 21$, which block the transition from G1 to $S$ phase, therefore repressing cell proliferation and cell cycle progression [52]. Furthermore, circFoxo3 can suppress the antisenescence and antistress roles of inhibitors of DNA binding, E2F transcription factor 1 (involved with regulation of retinoblastoma and glioblastoma multiforme), focal adhesion kinase, and hypoxiainducible factor- $1 \alpha$, by binding to them [53]. In addition, circRNAs can regulate the expression of linear proteinencoding RNA products by "mRNA trap" mechanisms.

\subsection{Regulation of Transcription or Alternative Splicing.} Recent advances have reported a cis-regulatory role of circRNAs on their parent coding genes (Figure 2(c)). CiRNA ankyrin repeat domain 52 (ci-ankrd52) largely accumulates in the elongating polymerase II (Pol II) complex and interacts with it to activate transcription of its parent gene. The expression of ANKRD52 mRNA was reduced when ci- ankrd52 was knocked down [41]. Subsequently, researchers also found EIciRNAs, such as circRNA eukaryotic translation initiation factor 3 subunit J (circEIF3J) and circRNA poly(A) binding protein interacting protein 2 (circPAIP2), predominantly localized in the nucleus. EIciRNAs can interact with the U1 small nuclear ribonucleoproteins (snRNPs) by specific RNA-RNA interactions between U snRNA and EIciRNAs, leading to increased interactions between the EIciRNA-U1 snRNP complexes with Pol II and enhancing transcription of their parental genes [42]. Additionally, another study uncovered that circRNAs can reduce protein expression by sequestering the translation start site and producing noncoding linear transcripts [39].

3.4. Protein Translation. Apart from the abovementioned functions, circRNAs are also involved in protein translation (Figure 2(d)). In 2017, it was confirmed that the endogenous circRNA zinc finger protein 609 (circZNF609) can encode proteins. CircZNF609 functions in murine and human myoblasts and can be translated into a protein in a splicingdependent and cap-independent way [54]. Pamudurti et al. demonstrated that circRNAs generated from the $M B L$ locus have protein-encoding capacity and allow cap-independent translation [34]. Furthermore, circRNA F-box and WD repeat domain containing 7 (circFBXW7) can translate short 


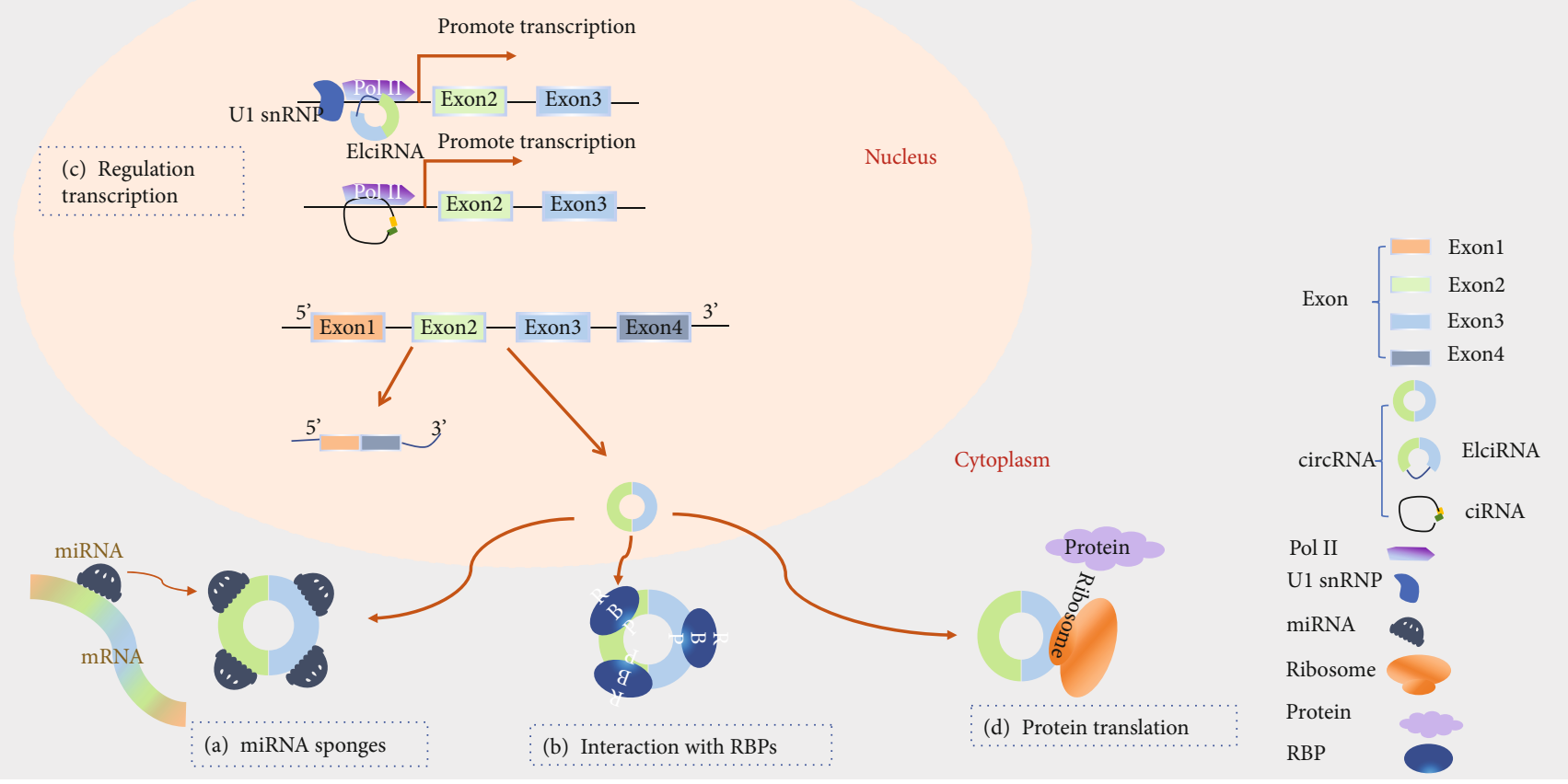

FIGURE 2: Schematic diagram illustrating functions of circRNAs. (a) Acting as miRNA sponges: circRNAs contain a common miRNA response element (MRE) that can bind to miRNA and prevent them from interacting with mRNA. (b) Interaction with RBPs: circRNAs can bind to RBPs to regulate mRNA expression by altering the splicing pattern or mRNA stability. (c) Regulation transcription: circRNAs play a regulatory role in the transcription of their parent coding genes. (d) Protein translation: circRNAs have coding potential and can be translated into proteins with ribosomes.

and stable FBXW7-185 amino acid, which is a novel protein playing a definitive tumor-suppressive role and has potential prognostic significance in brain cancer [55]. Evidence has also been found in neurological diseases that circSHPRH (SNF2 histone linker PHD RING helicase) encodes SHPRH, a novel protein of 146 amino acids, which extends patient survival time in glioblastoma patients [56].

\section{The Roles of circRNAs in Regulating Neurovascular Diseases}

The first study investigating the role of circRNAs in ischemic stroke was done by Mehta et al. in 2017. They found significant changes in the expression of circRNAs in the ischemic penumbral cortex in mice with transient middle cerebral artery occlusion (tMCAO). circRNA expression profiles in the penumbral cortex at 6,12 , and $24 \mathrm{~h}$ after reperfusion were investigated. There were 283 circRNAs altered in tMCAO mice compared with those in the sham group, and 16 of the 283 circRNAs changed at all three time points. These results indicated the possible functional implication of circRNAs in poststroke pathophysiology [57]. Dou et al. explored the expression of circRNAs in rat brains at 6,12 , and $24 \mathrm{~h}$ after the onset of intracerebral hemorrhage (ICH) using microarray analysis. There were 93 and 20 circRNAs that were upregulated and downregulated, respectively, at all times compared to those in the sham group. The authors designed a network of circRNA-miRNA-mRNA bioinformatic methods and found that circRNAs can reduce brain damage, attenuate neurological dysfunction, and improve prognosis after ICH, exposing a potential possibility of circRNAs as a therapeutic target for ICH [58]. In addition, Han et al. also observed the upregulation of circRNA HECT domain E3 ubiquitin-protein ligase 1 (circHECTD1) in the tMCAO mouse models by microarray analysis, which was validated in patients with acute ischemic stroke (AIS) [59].

CircHECTD1 was shown to serve as a microRNA-142 sponge to inhibit the expression of TCDD-inducible poly(ADP-ribose) polymerase expression, thus inhibiting the activation of astrocytes. Taken together, circHECTD1 also can be a novel biomarker of stroke [59]. CircDLGAP4 was increased in both a mouse stroke model and the plasmas of 13 female and 13 male patients [60]. It acts as an miRNA143 sponge, thereby inhibiting miR-143 activity and reducing HECTD1 expression. Importantly, the overexpression of circDLGAP4 protected nerves and alleviated blood-brain barrier damage in one study, making it a potential novel therapeutic target for the treatment of acute cerebral ischemia [60]. In China, researchers found 3 circRNAs (circFUNDC1, circPDS5B, and $\operatorname{circCDC14A)}$ which were significantly upregulated and positively correlated with infarct volume in the plasma of patients with AIS compared with healthy controls. The combination of these circRNAs has high specificity and sensitivity in the diagnosis of AIS [61]. As an important clinical pathological process, cerebral ischemia-reperfusion injury (IRI) is of great research value. 
Lin et al. established a model of oxygen-glucose deprivation/reoxygenation (OGD/R) in HT22 cells and compared the circRNA expression profile to normal controls by using circRNA microarrays. The results revealed the upregulation of 3 circRNAs and the downregulation of 15 circRNAs. By quantitative real-time PCR and bioinformatic analysis, a particular circRNA, mmu-circRNA-015947, was significantly upregulated and interacted with microRNAs to enhance target gene expression [62]. Another study analyzing the expression of circRNAs $24 \mathrm{~h}$ after reperfusion observed higher expression levels of circ_008018 in the cortical tissue of tMCAO mice than in the sham group. Notably, inhibition of circ_008018 can induce overexpression of miR-99a, thereby reversing the decrease of AKT phosphorylation and glycogen synthase kinase $3 \mathrm{~b}$ caused by IRI to protect brain tissue [63]. Atherosclerotic plaque rupture is an important pathogeny of AIS. In one study, circR-284, which has miR221 and miR-222 binding sites to regulate the activity of these miRNAs, was expressed in human vascular smooth muscle cells (VSMCs). These miRNAs inhibit oxidative stress induced by oxidized low-density lipoprotein and are novel regulators for VSMC proliferation and neointimal hyperplasia [64-66]. The expression of miR-221 and miR-222 decreases acutely, accompanied by an increase in p27Kip1 after plaque rupture, suggesting they may have plaquestabilizing capabilities [67]. Bazan et al. compared the expression of miR-221, miR-222, and circR-284 in serum between patients who suffered from an acutely symptomatic ischemic cerebrovascular event within the previous five days and asymptomatic patients. They found that the ratio of serum circR-284 to miR-221, which has the potential to detect plaque rupture and stroke, was significantly raised in the symptomatic group, and this result was verified in a study with 112 patients [64].

\section{Regulatory Role of CircRNAs in Oxidative Stress: Implications in Neurovascular Diseases}

ROS produced during oxidative stress causes oxidative damage to cells. ROS is increased in and associated with various risk factors of neurovascular diseases [13-16]. The roles of circRNAs in modulating oxidative stress have been discovered in both the basic and clinical studies (Table 1), and circRNAs are widely involved in different molecular mechanisms contributing to the pathophysiological processes of neurovascular disease $[12,68]$. Therefore, circRNAs may play an important role in the pathophysiology of neurovascular diseases.

5.1. CircRNAs Regulate Neurovascular Diseases and Other Vascular-Related Diseases through Modulating Oxidative Stress Processes. CircAkap7 was downregulated in a mouse model of tMCAO, which is a classic model for ischemic stroke. A high-throughput circRNA microarray study revealed that in tMCAO mice, mm9_circ_010383 (circAkap7) was suppressed [57]. A-kinase anchor protein 7 (AKAP7) is widely expressed throughout the brain, and the early heightened expression levels of AKAP7 were significantly associated with the blood-brain barrier [69, 70]. A study by $\mathrm{Xu}$ and colleagues reported that exo-circAkap7 can reverse the significantly high expression of ROS and malondialdehyde (MDA) in the tMCAO group. After the treatment with exo-circAkap7, the levels of circAkap7, autophagy-related gene ATG12 (Figure 3(a)), and oxidative stress-related gene $N R F 2$ were significantly increased via absorbing miR-155-5p [71] (Figure 3(b)). Up to now, there are a few papers showing the regulatory role of circRNAs in ischemic stroke through modulating oxidative stress. More studies should be designed to investigate this topic. Moreover, circRNAs have been found to be involved in vascularrelated diseases. CircRNA zinc finger protein 609 (circZNF609) was abundantly expressed in endothelial cells [72] and upregulated under hypoxia stress in a diabetic retinopathy mouse model [73]. Silencing circZNF609 protected endothelial cells from hypoxia and oxidative stress in vivo by reducing capillary degeneration and pathological angiogenesis, as well as partially rescuing human umbilical vein endothelial cells (HUVECs) from oxidative stress- and hypoxic stress-induced cell apoptosis [73]. Moreover, the overexpression of miR-615-5p can also reduce diabetes mellitusinduced retinal vascular leakage and rescue aggravated capillary degeneration from oxidative stress and hypoxia-induced apoptosis. Taken together, these results suggested circZNF609 works through a signaling network which is composed of circZNF609 and miR-615-5p [73]. Atherosclerosis is a pathological process of ischemic stroke [74]. Reducing the expression of circANRIL (circular antisense noncoding RNA in the INK4 locus) can prevent the progression of atherosclerosis by reducing the levels of inflammatory factors and the serum levels of lipids, triglycerides, lowdensity lipoprotein, interleukin- (IL-) 1, IL-6, matrix metalloproteinase-9, and C-reactive protein and alleviating the apoptosis of vascular endothelial cells [75]. Similarly, another study reported that circANRIL may alter RNA function and confer atheroprotection. It can also promote nucleolar stress and p53 activation by combining with the pescadillo ribosomal biogenesis factor 1 , thereby controlling the maturation of ribosomal RNA and inhibiting proliferation [9]. Shi et al. found that in patients with coronary heart disease, inhibiting circANRIL expression can reduce vascular endothelial injury, oxidative stress, and inflammation [76].

Liu et al. found that under oxidative stress, circHIPK3 (homeodomain interacting protein kinase 3) silencing increased the number of cell death, inhibited cell proliferation, and finally promoted the occurrence and development of apoptosis [77]. CircHIPK3 acts as a sponge for miR$193 a-3 p$, the inhibitor of which significantly increases the viability and proliferation and rescues the effects of circHIPK3 silencing. It is obvious that circHIPK3 regulates the function of human lens epithelial cells in vitro through the circHIPK3/miR-193a-3p/crystallin alpha A network axis [77]. In the cardiac microvascular endothelial cells (CMVECs) under oxidative stress conditions (hydrogen peroxide- $\left(\mathrm{H}_{2} \mathrm{O}_{2}^{-}\right)$induced), circHIPK 3 can be transferred by exosomes released from CMVECs pretreated with hypoxia and can regulate oxidative damage in these cells via the miR-29a/IGF-1 axis. circHIPK3 overexpression significantly decreases apoptosis and protects CMVECs against $\mathrm{H}_{2} \mathrm{O}_{2}$ - 


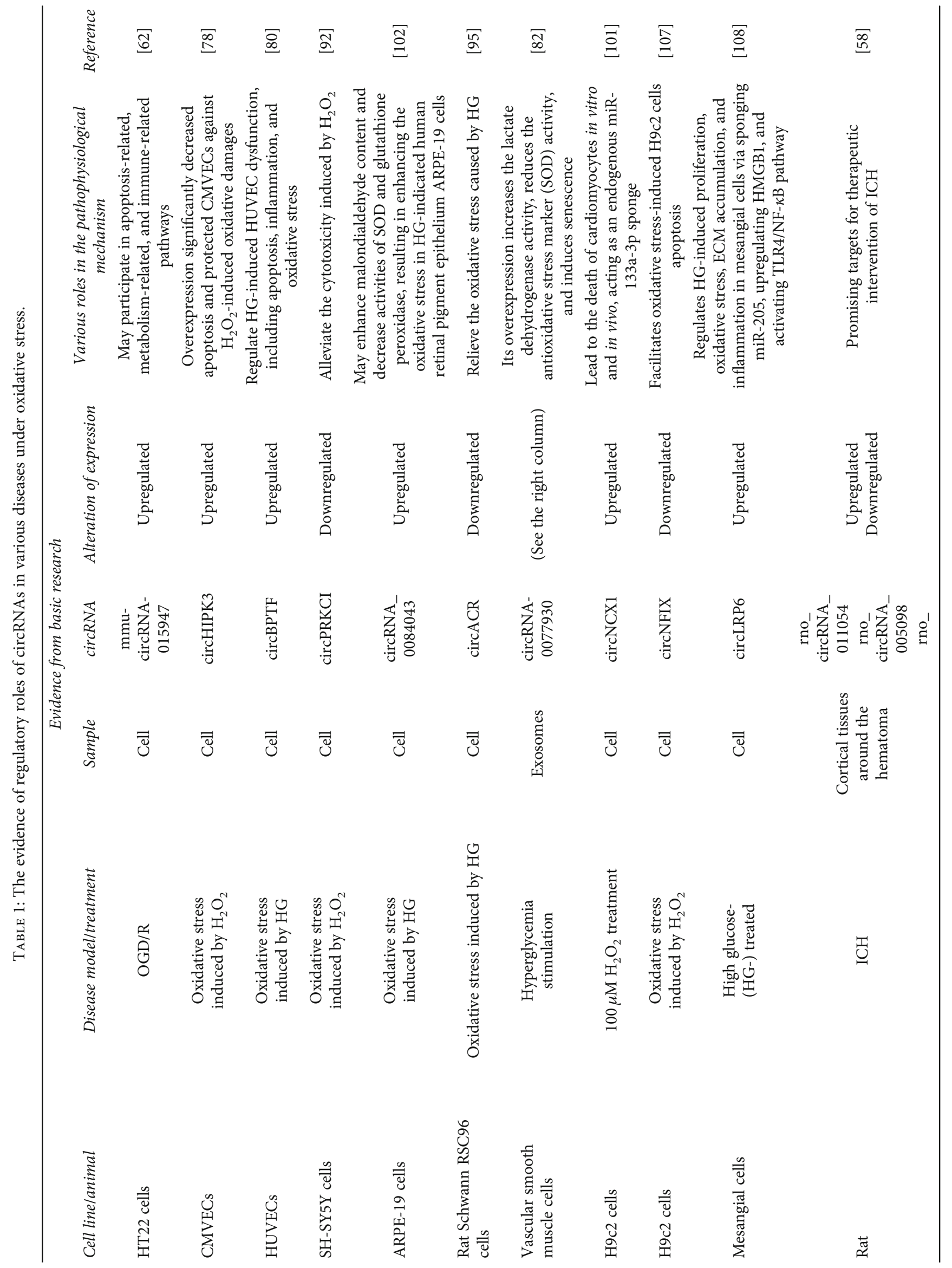




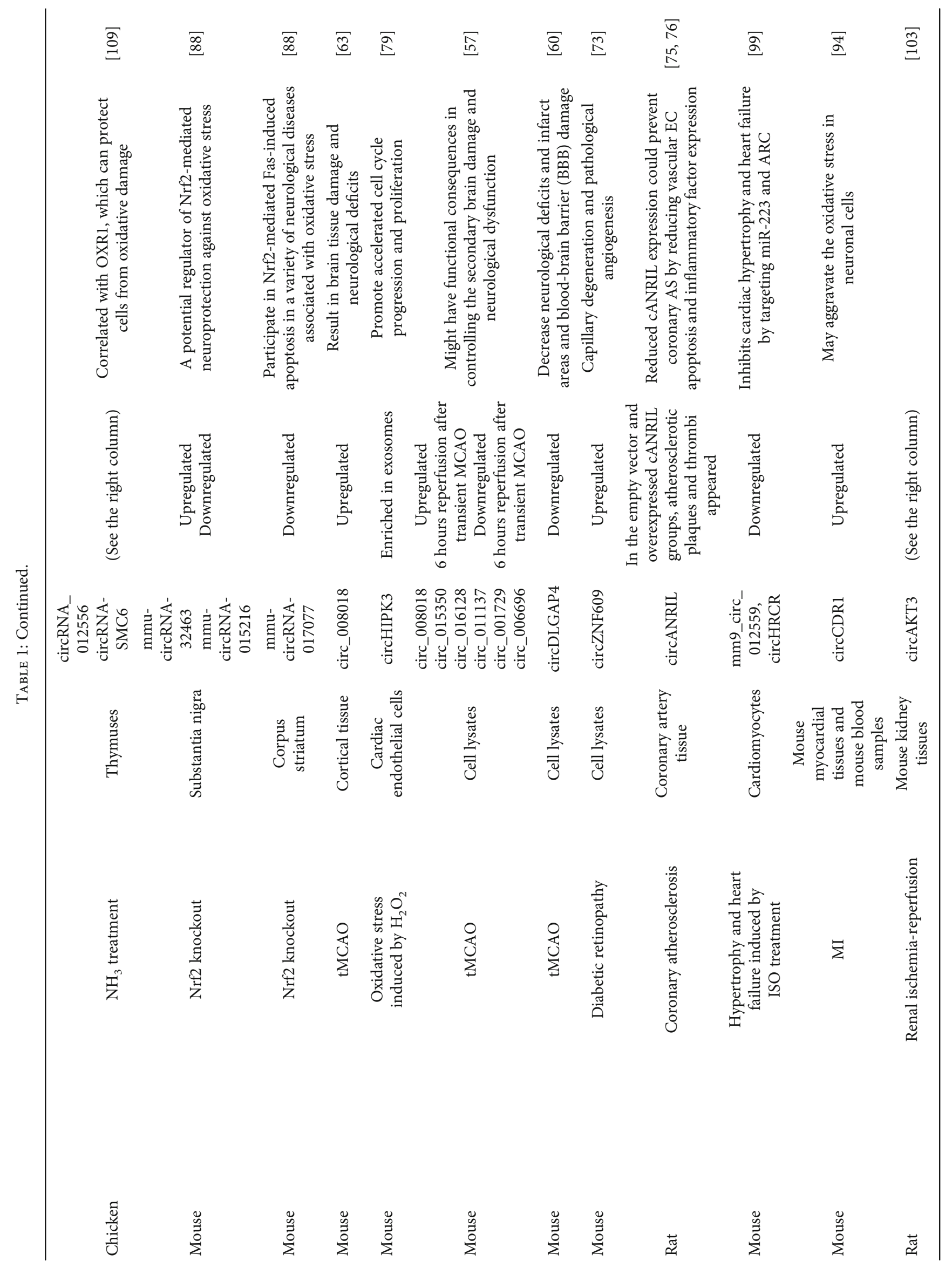




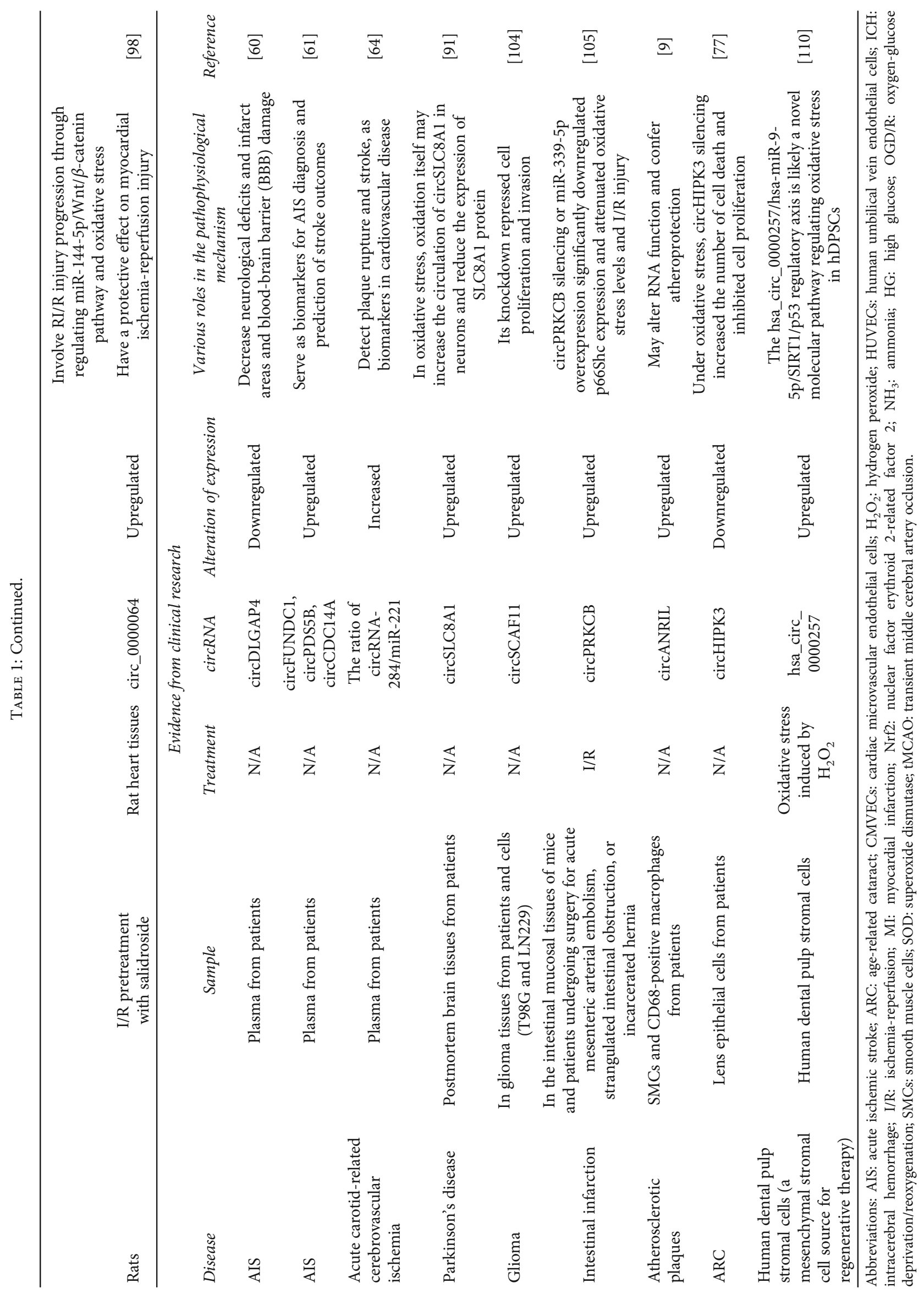




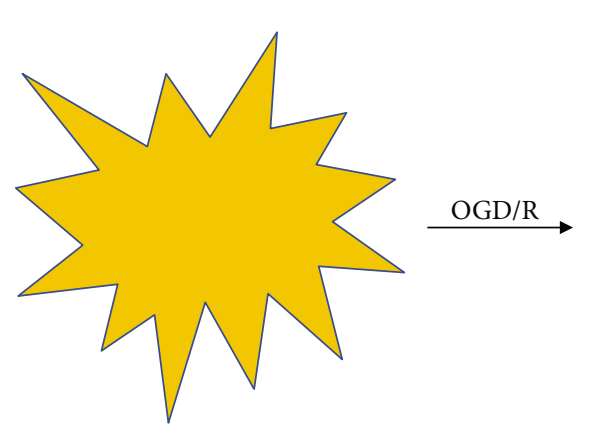

Healthy astrocyte

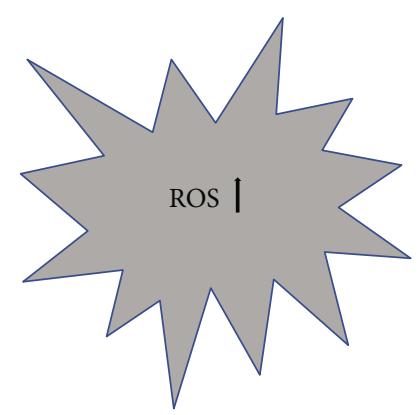

Injured astrocyte

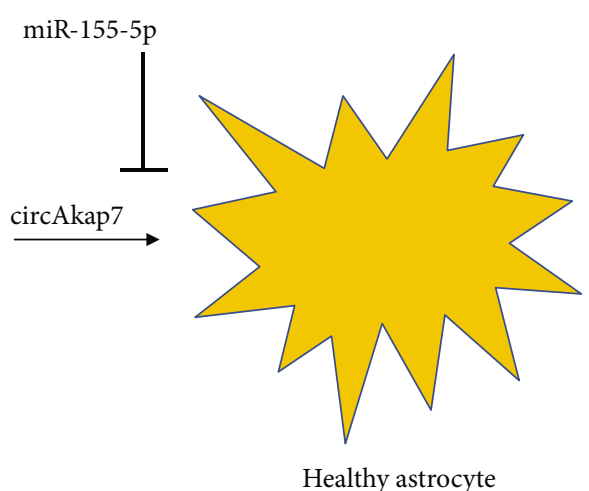

Healthy astrocyte

(a)
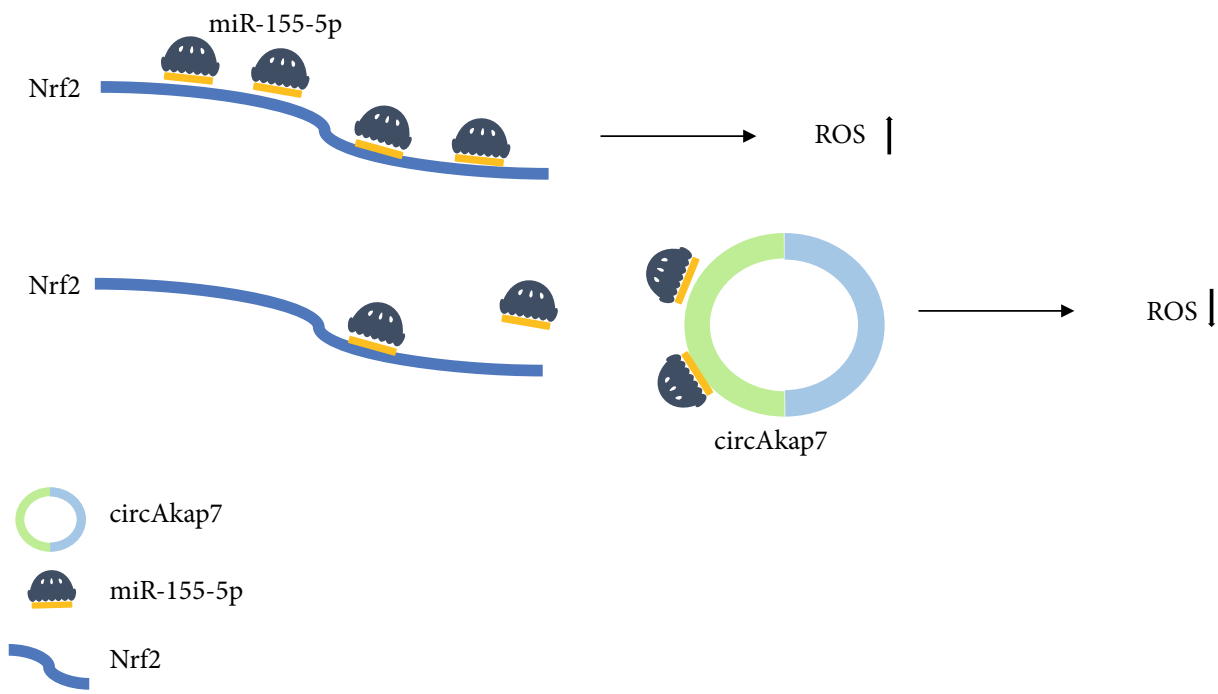

(b)

Figure 3: The regulatory role of circAkap7 via oxidative stress in a cell model of ischemic stroke. (a) The oxygen-glucose deprivation/reoxygenation (OGD/R) increases the reactive oxidative stress levels in primary astrocytes, whereas coculture with circAkap7 delivered by exosomes suppresses this increase. Moreover, circAkap7 is negatively regulated by miR-155-5p. (b) circAkap7 reduced OGD/R-induced cellular injury by absorbing miR-155-5p, which promotes Nrf2-mediated oxidative stress.

induced oxidative damage [78]. It is also reported that cardiac endothelial cells can internalize exosomes containing circHIPK3 and increase circHIPK3 levels causing inhibition of miR-29a activity, increasing VEGFA expression, and regulating angiogenesis [79]. In high glucose- (HG-) induced HUVECs, the expression of circBPTF (bromodomain PHD finger transcription factor) is upregulated. This regulates HG-induced HUVEC dysfunction, including apoptosis, inflammation, and oxidative stress through the miR384/LIN28B axis. The circBPTF knockdown can increase LIN28B to enhance cell viability and prevent inflammatory damage and oxidative stress [80]. LIN28B is an RNA binding protein which represses oncogenes by inhibiting the formation of the let-7 family of miRNAs [81]. A study by Wang and colleagues revealed that HG-induced HUVEC exosomes (HUVEC-Exos) treated VSMCs. Lactate dehydrogenase levels were higher, and the activity of the antioxidative stress marker, superoxide dismutase (SOD), was lower, compared with that of VSMCs incubated with normal glucose HUVEC-Exos. These results suggested that HG-HUVECExos with circRNA-0077930 can induce cellular oxidative stress and increase the expression of senescence-associated proteins (such as Kras, p21, and p53) via the circRNA0077930-miR-622-Kras ceRNA network, causing the senescence of VSMCs [82].

5.2. CircRNAs Regulate Other Neurological Diseases via Modulating Oxidative Stress Processes. Neural tissues are sensitive to oxidative stress, so oxidative stress is one of the major mechanisms involved in neuronal damage and death after AIS [83-85]. Nuclear factor erythroid 2-related factor 2 (Nrf2) is a transcription factor regulating the transcription of antioxidant response elements [86]. It drives the expression of various detoxification and antioxidant factors, such as SOD1, glutamate-cysteine ligase regulatory subunit, GPx2, and glutathione reductase through Nrf2-related pathways [87], thus playing a neuroprotective role in oxidative stress associated with acute neuronal injury and chronic neurodegeneration. Yang et al. found that mmu-circRNA-32463 and mmu-circRNA-015216 may be potential regulators of Nrf2-mediated neuroprotection against oxidative stress [88]. CircSLC8A1 (solute carrier family 8 member A1), 
arising from the sodium-calcium exchanger gene SLC8A1, is a circularized exon 2 transcript [89]. As an Argonaute-2bound RNA, it can also bind and may even modulate the activity of several miRNAs, including miR-128 and miR133a [90]. In oxidative stress, oxidation itself may increase the circulation of circSLC8A1 in neurons and reduce the degradation of circRNAs, ultimately reducing the expression of the SLC8A1 protein. Inversely, in human neuroblastoma SH-SY5Y cells treated with statins (drugs with antioxidant potential), both circSLC8A1 and SLC8A1 protein are reduced [91]. Cheng et al. show that $\mathrm{H}_{2} \mathrm{O}_{2}$ downregulates the expression of circPRKCI (protein kinase $\mathrm{C}$ iota) and induces neuronal injury through the circPRKCI-miR545/589-E2F2 axis. The overexpression of circPRKCI alleviates $\mathrm{H}_{2} \mathrm{O}_{2}$-induced cytotoxicity [92].

There are 15 circRNAs significantly changed in an oxygen-glucose deprivation/reoxygenation (OGD/R) model of cultured hippocampal HT22 cells, and the upregulated expression of mmu-circRNA-015947 indicated that it may be involved in the pathogenesis of cerebral IRI through the Kyoto Encyclopedia of Genes and Genomes pathway analysis [62]. Ding et al. found that the upregulation of miR-7a could regulate mitochondrial function and enhance the expression of antioxidases to alleviate the injury-induced oxidative stress in rats with spinal cord injuries [93]. CircRNA Cdrlas is derived from an antisense transcript of the $C d r 1$ proteincoding gene at chromosome $\mathrm{X}$ (NC_000086.7) in mice [94] and functions as an miR-7 sponge in neuronal cells [7]; therefore, it can be speculated that circCDR1 may aggravate the oxidative stress in neuronal cells by acting as an miR-7 sponge [94].

Liu et al. discovered that the expression of circACR was reduced in HG-irritated rat Schwann RSC96 cells, which relieved the oxidative stress caused by $\mathrm{HG}$ via declining miR-145-3p and promoting PI3K/AKT/mTOR pathway activation [95].

\subsection{CircRNAs Regulate Various Diseases via Modulating} Oxidative Stress Processes. Apart from neurological diseases, regulatory roles of circRNAs through modulating oxidative stress are also found in other diseases, which are summarized in Table 1.

5.3.1. Cardiomyocytes. Tian et al. found that atorvastatin can rescue cardiomyocytes from oxidative stress-induced apoptosis through decreasing microRNA-143 (miR-143) levels [96], and Bai et al. demonstrated that circDLGAP4 can inhibit miR-143 activity by acting as an endogenous miR143 sponge [60]. In another study, Wang et al. found that the circDLGAP4/miR-143/BCL2 axis is a potential regulated axis in oxidative stress and myocardial IRI [97]. Upregulation of hsa_circ_0000064 (circ_0000064) weakened autophagy, reduced levels of lactate creatine kinase and dehydrogenase, inhibited oxidative stress, and significantly reduced myocardial infarction areas, signaling circ_0000064 had a protective effect against myocardial IRI [98]. Wang et al. found that acting as an miR-223 sponge, the heart-related circRNA could sequester and inhibit the activity of miR-223, which is confirmed as an important regulator of cardiomyocyte apoptosis under oxidative stress $[99,100]$. In H9c2 (fetal cardiomyocyte-derived) cells, upon treatment with $100 \mu \mathrm{M} \mathrm{H}_{2} \mathrm{O}_{2}$, it was observed that the circNCX1 level increased. Acting as an endogenous miR-133a-3p sponge, circNCX1 responds to the ROS increase which eventually leads to the expression of proapoptotic factor cell death-inducing protein, which ultimately leads to the death of cardiomyocytes in vitro and in vivo [101].

5.3.2. Others. Li et al. reported that the knockdown of circRNA_0084043 may decrease MDA content and enhance activities of SOD and glutathione peroxidase, resulting in weakening the oxidative stress in HG-indicated human retinal pigment epithelium ARPE-19 cells [102]. It has been established that circAKT3 can regulate oxidative stress to promote renal IRI progression. In I/R+circAKT3 rats, the MDA and $\mathrm{O}_{2}$ levels were significantly increased and the SOD and catalase levels were significantly downregulated [103]. In the experiments performed by Yin et al., the knockdown of circSCAF11 upregulated cellular ROS levels and promoted 8-hydroxy-2-deoxyguanosine (8-OHdG) production in T98G and LN229 cells, which can be decreased by an miR-145-5p inhibitor [104].

Feng et al. revealed a crucial role for the circPRKCB $/ \mathrm{miR}$ $339-5 \mathrm{p} / \mathrm{p} 66 \mathrm{Shc}$ signaling pathway in regulating oxidative stress in the I/R intestine. CircPRKCB can regulate p66Shc expression and hypoxia/reoxygenation- (H/R-) induced oxidative stress acting as an endogenous miR-339-5p sponge. The silencing of circPRKCB significantly inhibited the increased expression of intracellular ROS levels, mitochondrial $\mathrm{O}_{2}$ levels, and NOX2 and the decreased expression of $\mathrm{H} / \mathrm{R}$-induced manganese SOD and catalase [105].

Researchers found that in $\mathrm{HaCaT}$ cells treated with $\mathrm{H}_{2} \mathrm{O}_{2}$, which were considered a cell model of pressure ulcers, circZNF609 silencing may reduce oxidative stress by regulating miR-145 [106]. CircNFIX is abundant, conservative, and stable in H9c2 cells. In an anoxic environment, circNFIX expression was significantly downregulated in cardiomyocytes under oxidative stress [107]. Chen and colleagues found that the HG treatment can significantly increase ROS and MDA levels and decrease SOD activity and SOD2 expression. Moreover, they reported that circLRP6 regulates HGinduced proliferation, extracellular matrix accumulation, inflammation in mesangial cells, and oxidative stress via sponging miR-205, upregulating high mobility group box 1 , and activating toll-like receptor 4/nuclear factor-kappa B signaling [108].

\section{Perspectives}

CircRNAs are potential pivotal biomarkers for diagnosis of diseases and prognosis because they are abundant, stable, and conserved. CircRNAs participate in regulating gene expression in various diseases through three molecular mechanisms: acting as a sponge of miRNAs or ceRNAs, interaction with RBPs and mRNAs, and regulation of transcription or alternative splicing. Increasingly, there is evidence to show that circRNAs are involved in the process of oxidative stress, which has been shown to be closely related 
to neurovascular diseases. Therefore, circRNAs may affect the occurrence and development of neurovascular diseases by regulating the oxidative stress process. In this review, the possible changes in circRNAs in cerebrovascular diseases and the results were described in detail, suggesting that circRNAs may be a biomarker of cerebrovascular diseases and a potential new therapeutic target. This article also summarizes that circRNAs regulate the occurrence and development of neurovascular diseases and other vascular-related diseases by regulating oxidative stress: first, under oxidative stress, circRNAs change and result in the development of diseases; second, the change in circRNAs may lead to oxidative stress and result in the occurrence and development of diseases. All evidence suggests that circRNAs may be important in the oxidative stress-induced pathophysiology of neurovascular diseases.

\section{Abbreviations}

\begin{tabular}{|c|c|}
\hline AIS: & Acute ischemic stroke \\
\hline ankrd52: & Ankyrin repeat domain 52 \\
\hline ANRIL: & $\begin{array}{l}\text { Antisense noncoding RNA in the INK4 } \\
\text { locus }\end{array}$ \\
\hline BPTF: & $\begin{array}{l}\text { Bromodomain PHD finger transcription } \\
\text { factor }\end{array}$ \\
\hline ceRNAs: & Competitive endogenous RNAs \\
\hline circRNAs: & Circular RNAs \\
\hline ciRNAs: & Circular intronic RNAs \\
\hline CMVECs: & Cardiac microvascular endothelial cells \\
\hline DLGAP4: & DLG-associated protein 4 \\
\hline ecircRNA: & Exonic circRNA \\
\hline ecircRNAs: & Exonic circular RNAs \\
\hline EIciRNAs: & Exon-intron circular RNAs \\
\hline FBXW7: & F-box and WD repeat domain containing 7 \\
\hline Foxo3: & Forkhead box O3 \\
\hline GPx: & Glutathione peroxidase \\
\hline $\mathrm{H}_{2} \mathrm{O}_{2}:$ & Hydrogen peroxide \\
\hline HECTD1: & HECT domain E3 ubiquitin-protein ligase 1 \\
\hline HG: & High glucose \\
\hline HIPK3: & Homeodomain interacting protein kinase 3 \\
\hline H/R: & Hypoxia/reoxygenation \\
\hline HUVEC: & Human umbilical vein endothelial cell \\
\hline HUVEC-Exos: & $\begin{array}{l}\text { Human umbilical vein endothelial cell } \\
\text { exosomes }\end{array}$ \\
\hline ICH: & Intracerebral hemorrhage \\
\hline I/R: & Ischemia-reperfusion \\
\hline IRI: & Ischemia-reperfusion injury \\
\hline ITCH: & Itchy E3 ubiquitin-protein ligase \\
\hline lncRNAs: & Long noncoding RNAs \\
\hline MBL: & Muscleblind \\
\hline miRNAs: & MicroRNAs \\
\hline Nrf2: & Nuclear factor erythroid 2-related factor 2 \\
\hline${ }^{1} \mathrm{O}_{2}:$ & Singlet oxygen \\
\hline $\mathrm{O}_{2}^{-}:$ & Superoxide anion \\
\hline $\mathrm{O}_{3}:$ & Ozone \\
\hline OGD/R: & Oxygen-glucose deprivation/reoxygenation \\
\hline $\mathrm{OH}^{-}:$ & Hydroxyl radicals \\
\hline OXR1: & Antioxidant 1 \\
\hline PABPN1: & Poly(A) binding protein nuclear 1 \\
\hline
\end{tabular}

PRKCI: $\quad$ Protein kinase C iota

RBPs: $\quad$ RNA binding proteins

ROS: $\quad$ Reactive oxygen species

SHPRH: $\quad$ SNF2 histone linker PHD RING helicase

SH-SY: Human neuroblastoma

SLC8A1: $\quad$ Solute carrier family 8 member A1

snRNPs: $\quad$ Small nuclear ribonucleoproteins

SOD: $\quad$ Superoxide dismutase

tMCAO: $\quad$ Transient middle cerebral artery occlusion

VSMCs: $\quad$ Vascular smooth muscle cells

ZNF609: $\quad$ Zinc finger protein 609.

\section{Conflicts of Interest}

All authors state that there is no conflict of interest.

\section{Authors' Contributions}

Lingfei Li, Zhumei Ni, and Xiaoli Si have contributed equally to this work.

\section{Acknowledgments}

This work was funded by the National Natural Science Foundation of China (81801157), Zhejiang Basic Public Welfare Research Program (LGF20H090008), and Hangzhou Health Science and Technology Project (ZD20200056).

\section{References}

[1] L. S. Kristensen, M. S. Andersen, L. V. W. Stagsted, K. K. Ebbesen, T. B. Hansen, and J. Kjems, "The biogenesis, biology and characterization of circular RNAs," Nature Reviews. Genetics, vol. 20, no. 11, pp. 675-691, 2019.

[2] N. Eger, L. Schoppe, S. Schuster, U. Laufs, and J. N. Boeckel, "Circular RNA splicing," Advances in Experimental Medicine and Biology, vol. 1087, pp. 41-52, 2018.

[3] W. R. Jeck, J. A. Sorrentino, K. Wang et al., "Circular RNAs are abundant, conserved, and associated with ALU repeats," RNA, vol. 19, no. 2, pp. 141-157, 2013.

[4] B. Han, J. Chao, and H. Yao, "Circular RNA and its mechanisms in disease: from the bench to the clinic," Pharmacology \& Therapeutics, vol. 187, pp. 31-44, 2018.

[5] J. Salzman, C. Gawad, P. L. Wang, N. Lacayo, and P. O. Brown, "Circular RNAs are the predominant transcript isoform from hundreds of human genes in diverse cell types," PLoS One, vol. 7, no. 2, article e30733, 2012.

[6] S. Memczak, M. Jens, A. Elefsinioti et al., "Circular RNAs are a large class of animal RNAs with regulatory potency," Nature, vol. 495, no. 7441, pp. 333-338, 2013.

[7] T. B. Hansen, T. I. Jensen, B. H. Clausen et al., "Natural RNA circles function as efficient microRNA sponges," Nature, vol. 495, no. 7441, pp. 384-388, 2013.

[8] M. Zhou, H. Wang, X. Zeng et al., "Mortality, morbidity, and risk factors in China and its provinces, 1990-2017: a systematic analysis for the Global Burden of Disease Study 2017," Lancet, vol. 394, no. 10204, pp. 1145-1158, 2019.

[9] L. M. Holdt, A. Stahringer, K. Sass et al., "Circular noncoding RNA_ANRIL_ modulates ribosomal RNA maturation and atherosclerosis in humans," Nature Communications, vol. 7, no. 1, article 12429, 2016. 
[10] L. Shen, Y. Bai, B. Han, and H. Yao, "Non-coding RNA and neuroinflammation: implications for the therapy of stroke," Stroke Vasc Neurol, vol. 4, no. 2, pp. 96-98, 2019.

[11] A. Rybak-Wolf, C. Stottmeister, P. Glažar et al., "Circular RNAs in the mammalian brain are highly abundant, conserved, and dynamically expressed," Molecular Cell, vol. 58, no. 5, pp. 870-885, 2015.

[12] X. Zhang, M. H. Hamblin, and K. J. Yin, "Noncoding RNAs and stroke," The Neuroscientist, vol. 25, no. 1, pp. 22-26, 2019.

[13] A. Morsch, E. Wisniewski, T. F. Luciano et al., "Cigarette smoke exposure induces ROS-mediated autophagy by regulating sestrin, AMPK, and mTOR level in mice," Redox Report, vol. 24, no. 1, pp. 27-33, 2019.

[14] N. Wu, H. Shen, H. Liu, Y. Wang, Y. Bai, and P. Han, “Acute blood glucose fluctuation enhances rat aorta endothelial cell apoptosis, oxidative stress and pro-inflammatory cytokine expression in vivo," Cardiovascular Diabetology, vol. 15, no. 1, p. 109, 2016.

[15] N. Sinha and P. K. Dabla, "Oxidative stress and antioxidants in hypertension-a current review," Current Hypertension Reviews, vol. 11, no. 2, pp. 132-142, 2015.

[16] M. Petráš, A. Drgová, M. Kovalská et al., "Effect of hyperhomocysteinemia on redox balance and redox defence enzymes in ischemia-reperfusion injury and/or after ischemic preconditioning in rats," Cellular and Molecular Neurobiology, vol. 37, no. 8, pp. 1417-1431, 2017.

[17] P. Fuschi, B. Maimone, C. Gaetano, and F. Martelli, "Noncoding RNAs in the vascular system response to oxidative stress," Antioxidants \& Redox Signaling, vol. 30, no. 7, pp. 992-1010, 2019.

[18] M. T. Hsu and M. Coca-Prados, "Electron microscopic evidence for the circular form of RNA in the cytoplasm of eukaryotic cells," Nature, vol. 280, no. 5720, pp. 339-340, 1979.

[19] H. L. Sanger, G. Klotz, D. Riesner, H. J. Gross, and A. K. Kleinschmidt, "Viroids are single-stranded covalently closed circular RNA molecules existing as highly base-paired rodlike structures," Proceedings of the National Academy of Sciences of the United States of America, vol. 73, no. 11, pp. 3852-3856, 1976.

[20] B. Capel, A. Swain, S. Nicolis et al., "Circular transcripts of the testis-determining gene_Sry_ in adult mouse testis," Cell, vol. 73, no. 5, pp. 1019-1030, 1993.

[21] C. Cocquerelle, B. Mascrez, D. Hétuin, and B. Bailleul, "Missplicing yields circular RNA molecules," The FASEB Journal, vol. 7, no. 1, pp. 155-160, 1993.

[22] J. O. Westholm, P. Miura, S. Olson et al., "Genome-wide analysis of _Drosophila_circular RNAs reveals their structural and sequence properties and age-dependent neural accumulation," Cell Reports, vol. 9, no. 5, pp. 1966-1980, 2014.

[23] A. Ivanov, S. Memczak, E. Wyler et al., "Analysis of intron sequences reveals hallmarks of circular RNA biogenesis in animals," Cell Reports, vol. 10, no. 2, pp. 170-177, 2015.

[24] R. Dong, X. K. Ma, L. L. Chen, and L. Yang, "Increased complexity of circRNA expression during species evolution," RNA Biology, vol. 14, no. 8, pp. 1064-1074, 2017.

[25] T. Lu, L. Cui, Y. Zhou et al., "Transcriptome-wide investigation of circular RNAs in rice," RNA, vol. 21, no. 12, pp. 2076-2087, 2015.
[26] C. Y. Ye, L. Chen, C. Liu, Q. H. Zhu, and L. Fan, "Widespread noncoding circular RNAs in plants," The New Phytologist, vol. 208, no. 1, pp. 88-95, 2015.

[27] S. P. Barrett, P. L. Wang, and J. Salzman, "Circular RNA biogenesis can proceed through an exon-containing lariat precursor," eLife, vol. 4, article e07540, 2015.

[28] K. M. Broadbent, J. C. Broadbent, U. Ribacke, D. Wirth, J. L. Rinn, and P. C. Sabeti, "Strand-specific RNA sequencing in Plasmodium falciparum malaria identifies developmentally regulated long non-coding RNA and circular RNA," BMC Genomics, vol. 16, no. 1, p. 454, 2015.

[29] P. L. Wang, Y. Bao, M. C. Yee et al., "Circular RNA is expressed across the eukaryotic tree of life," PLoS One, vol. 9, no. 3, article e90859, 2014.

[30] H. Suzuki, Y. Zuo, J. Wang, M. Q. Zhang, A. Malhotra, and A. Mayeda, "Characterization of RNase R-digested cellular RNA source that consists of lariat and circular RNAs from pre-mRNA splicing," Nucleic Acids Research, vol. 34, no. 8, article e63, 2006.

[31] H. Suzuki and T. Tsukahara, "A view of pre-mRNA splicing from RNase R resistant RNAs,” International Journal of Molecular Sciences, vol. 15, no. 6, pp. 9331-9342, 2014.

[32] J. Salzman, R. E. Chen, M. N. Olsen, P. L. Wang, and P. O. Brown, "Cell-type specific features of circular RNA expression," PLoS Genetics, vol. 9, no. 9, article e1003777, 2013.

[33] J. U. Guo, V. Agarwal, H. Guo, and D. P. Bartel, "Expanded identification and characterization of mammalian circular RNAs," Genome Biology, vol. 15, no. 7, p. 409, 2014.

[34] N. R. Pamudurti, O. Bartok, M. Jens et al., "Translation of circRNAs,” Molecular Cell, vol. 66, no. 1, pp. 9-21.e7, 2017, e7.

[35] S. P. Barrett and J. Salzman, "Circular RNAs: analysis, expression and potential functions," Development, vol. 143, no. 11, pp. 1838-1847, 2016.

[36] W. Chen and E. Schuman, "Circular RNAs in brain and other tissues: a functional enigma," Trends in Neurosciences, vol. 39, no. 9, pp. 597-604, 2016.

[37] X. Li, L. Yang, and L. L. Chen, "The biogenesis, functions, and challenges of circular RNAs," Molecular Cell, vol. 71, no. 3, pp. 428-442, 2018.

[38] S. Guil and M. Esteller, "RNA-RNA interactions in gene regulation: the coding and noncoding players," Trends in Biochemical Sciences, vol. 40, no. 5, pp. 248-256, 2015.

[39] W. R. Jeck and N. E. Sharpless, "Detecting and characterizing circular RNAs," Nature Biotechnology, vol. 32, no. 5, pp. 453461, 2014.

[40] L. L. Chen and L. Yang, "Regulation of circRNA biogenesis," RNA Biology, vol. 12, no. 4, pp. 381-388, 2015.

[41] Y. Zhang, X. O. Zhang, T. Chen et al., "Circular intronic long noncoding RNAs," Molecular Cell, vol. 51, no. 6, pp. 792-806, 2013.

[42] Z. Li, C. Huang, C. Bao et al., "Exon-intron circular RNAs regulate transcription in the nucleus," Nature Structural \& Molecular Biology, vol. 22, no. 3, pp. 256-264, 2015.

[43] X. O. Zhang, H. B. Wang, Y. Zhang, X. Lu, L. L. Chen, and L. Yang, "Complementary sequence-mediated exon circularization," Cell, vol. 159, no. 1, pp. 134-147, 2014.

[44] L. L. Chen, "The biogenesis and emerging roles of circular RNAs," Nature Reviews. Molecular Cell Biology, vol. 17, no. 4, pp. 205-211, 2016.

[45] E. Junn, K. W. Lee, B. S. Jeong, T. W. Chan, J. Y. Im, and M. M. Mouradian, "Repression of alpha-synuclein expression 
and toxicity by microRNA-7," Proceedings of the National Academy of Sciences of the United States of America, vol. 106, no. 31, pp. 13052-13057, 2009.

[46] Y. Wang, J. Liu, C. Liu, A. Naji, and D. A. Stoffers, "MicroRNA-7 regulates the mTOR pathway and proliferation in adult pancreatic -cells," Diabetes, vol. 62, no. 3, pp. 887895, 2013.

[47] G. Militello, T. Weirick, D. John, C. Döring, S. Dimmeler, and S. Uchida, "Screening and validation of lncRNAs and circRNAs as miRNA sponges," Briefings in Bioinformatics, vol. 18, no. 5, pp. 780-788, 2017.

[48] T. B. Hansen, E. D. Wiklund, J. B. Bramsen et al., "miRNAdependent gene silencing involving Ago2-mediated cleavage of a circular antisense RNA," The EMBO Journal, vol. 30, no. 21, pp. 4414-4422, 2011.

[49] R. Ashwal-Fluss, M. Meyer, N. R. Pamudurti et al., "circRNA biogenesis competes with pre-mRNA splicing," Molecular Cell, vol. 56, no. 1, pp. 55-66, 2014.

[50] K. Abdelmohsen, A. C. Panda, R. Munk et al., "Identification of HuR target circular RNAs uncovers suppression of PABPN1 translation by circPABPN1," RNA Biology, vol. 14, no. 3, pp. 361-369, 2017.

[51] Z. G. Yang, F. M. Awan, W. W. du et al., "The circular RNA interacts with STAT3, increasing its nuclear translocation and wound repair by modulating Dnmt3a and miR-17 function," Molecular Therapy, vol. 25, no. 9, pp. 2062-2074, 2017.

[52] W. W. du, W. Yang, E. Liu, Z. Yang, P. Dhaliwal, and B. B. Yang, "Foxo3 circular RNA retards cell cycle progression via forming ternary complexes with p21 and CDK2," Nucleic Acids Research, vol. 44, no. 6, pp. 2846-2858, 2016.

[53] W. W. du, W. Yang, Y. Chen et al., "Foxo3 circular RNA promotes cardiac senescence by modulating multiple factors associated with stress and senescence responses," European Heart Journal, vol. 38, no. 18, pp. 1402-1412, 2017.

[54] I. Legnini, G. di Timoteo, F. Rossi et al., "circ-ZNF609 is a circular RNA that can be translated and functions in myogenesis," Molecular Cell, vol. 66, no. 1, pp. 22-37.e9, 2017, e9.

[55] Y. Yang, X. Gao, M. Zhang et al., "Novel role of FBXW7 circular RNA in repressing glioma tumorigenesis," Journal of the National Cancer Institute, vol. 110, no. 3, pp. 304-315, 2018.

[56] S. Begum, A. Yiu, J. Stebbing, and L. Castellano, "Novel tumour suppressive protein encoded by circular RNA, _circ-SHPRH_, in glioblastomas," Oncogene, vol. 37, no. 30, pp. 4055-4057, 2018.

[57] S. L. Mehta, G. Pandi, and R. Vemuganti, "Circular RNA expression profiles alter significantly in mouse brain after transient focal ischemia," Stroke, vol. 48, no. 9, pp. 25412548, 2017.

[58] Z. Dou, Q. Yu, G. Wang et al., "Circular RNA expression profiles alter significantly after intracerebral hemorrhage in rats," Brain Research, vol. 1726, article 146490, 2020.

[59] B. Han, Y. Zhang, Y. Zhang et al., "Novel insight into circular RNA HECTD1 in astrocyte activation via autophagy by targeting MIR142-TIPARP: implications for cerebral ischemic stroke," Autophagy, vol. 14, no. 7, pp. 1164-1184, 2018.

[60] Y. Bai, Y. Zhang, B. Han et al., "Circular RNA DLGAP4 ameliorates ischemic stroke outcomes by targeting miR-143 to regulate endothelial-mesenchymal transition associated with blood-brain barrier integrity," The Journal of Neuroscience, vol. 38 , no. 1, pp. 32-50, 2018.
[61] L. Zuo, L. Zhang, J. Zu et al., "Circulating circular RNAs as biomarkers for the diagnosis and prediction of outcomes in acute ischemic stroke," Stroke, vol. 51, no. 1, pp. 319-323, 2020.

[62] S. P. Lin, S. Ye, Y. Long et al., "Circular RNA expression alterations are involved in OGD/R-induced neuron injury," Biochemical and Biophysical Research Communications, vol. 471, no. 1, pp. 52-56, 2016.

[63] X. Yang, H. Ji, Y. Yao et al., "Downregulation of circ_008018 protects against cerebral ischemia-reperfusion injury by targeting miR-99a," Biochemical and Biophysical Research Communications, vol. 499, no. 4, pp. 758-764, 2018.

[64] H. A. Bazan, S. A. Hatfield, A. Brug, A. J. Brooks, D. J. Lightell Jr., and T. C. Woods, "Carotid plaque rupture is accompanied by an increase in the ratio of serum circR-284 to miR-221 levels," Circulation Cardiovascular Genetics, vol. 10, no. 4, 2017.

[65] X. Liu, Y. Cheng, S. Zhang, Y. Lin, J. Yang, and C. Zhang, “A necessary role of miR-221 and miR-222 in vascular smooth muscle cell proliferation and neointimal hyperplasia," Circulation Research, vol. 104, no. 4, pp. 476-487, 2009.

[66] X. Zhuang, R. Li, A. Maimaitijiang et al., "miR-221-3p inhibits oxidized low-density lipoprotein induced oxidative stress and apoptosis via targeting a disintegrin and metalloprotease-22," Journal of Cellular Biochemistry, vol. 120, no. 4, pp. 6304-6314, 2019.

[67] X. Liu, Y. Cheng, J. Yang, L. Xu, and C. Zhang, "Cell-specific effects of miR-221/222 in vessels: molecular mechanism and therapeutic application," Journal of Molecular and Cellular Cardiology, vol. 52, no. 1, pp. 245-255, 2012.

[68] A. J. Kattoor, N. V. K. Pothineni, D. Palagiri, and J. L. Mehta, "Oxidative stress in atherosclerosis," Current Atherosclerosis Reports, vol. 19, no. 11, p. 42, 2017.

[69] B. W. Jones, J. Deem, T. J. Younts et al., "Targeted deletion of AKAP7 in dentate granule cells impairs spatial discrimination," eLife, vol. 5, 2016.

[70] G. C. O'Connell, M. B. Treadway, A. B. Petrone et al., "Peripheral blood AKAP7 expression as an early marker for lymphocyte-mediated post-stroke blood brain barrier disruption," Scientific Reports, vol. 7, no. 1, p. 1172, 2017.

[71] L. Xu, H. Ji, Y. Jiang et al., "Exosomes derived from circAkap7-modified adipose-derived mesenchymal stem cells protect against cerebral ischemic injury," Frontiers in Cell and Development Biology, vol. 8, article 569977, 2020.

[72] J. N. Boeckel, N. Jaé, A. W. Heumüller et al., "Identification and characterization of hypoxia-regulated endothelial circular RNA," Circulation Research, vol. 117, no. 10, pp. 884890, 2015.

[73] C. Liu, M. D. Yao, C. P. Li et al., "Silencing of circular RNAZNF609 ameliorates vascular endothelial dysfunction," Theranostics, vol. 7, no. 11, pp. 2863-2877, 2017.

[74] W. Herrington, B. Lacey, P. Sherliker, J. Armitage, and S. Lewington, "Epidemiology of atherosclerosis and the potential to reduce the global burden of atherothrombotic disease," Circulation Research, vol. 118, no. 4, pp. 535-546, 2016.

[75] C. L. Song, J. P. Wang, X. Xue et al., "Effect of circular ANRIL on the inflammatory response of vascular endothelial cells in a rat model of coronary atherosclerosis," Cellular Physiology and Biochemistry, vol. 42, no. 3, pp. 1202-1212, 2017. 
[76] P. Shi, H. Ji, H. Zhang, J. Yang, R. Guo, and J. Wang, "circANRIL reduces vascular endothelial injury, oxidative stress and inflammation in rats with coronary atherosclerosis," Experimental and Therapeutic Medicine, vol. 20, no. 3, pp. 2245-2251, 2020.

[77] X. Liu, B. Liu, M. Zhou et al., "Circular RNA HIPK3 regulates human lens epithelial cells proliferation and apoptosis by targeting the miR-193a/CRYAA axis," Biochemical and Biophysical Research Communications, vol. 503, no. 4, pp. 22772285, 2018.

[78] Y. Wang, R. Zhao, W. Liu et al., "Exosomal circHIPK3 released from hypoxia-pretreated cardiomyocytes regulates oxidative damage in cardiac microvascular endothelial cells via the miR-29a/IGF-1 pathway," Oxidative Medicine and Cellular Longevity, vol. 2019, Article ID 7954657, 28 pages, 2019.

[79] Y. Wang, R. Zhao, C. Shen et al., "Exosomal circHIPK3 released from hypoxia-induced cardiomyocytes regulates cardiac angiogenesis after myocardial infarction," Oxidative Medicine and Cellular Longevity, vol. 2020, Article ID 8418407, 19 pages, 2020.

[80] W. Zhang and Y. Sui, "circBPTF knockdown ameliorates high glucose-induced inflammatory injuries and oxidative stress by targeting the miR-384/LIN28B axis in human umbilical vein endothelial cells," Molecular and Cellular Biochemistry, vol. 471, no. 1-2, pp. 101-111, 2020.

[81] J. Balzeau, M. R. Menezes, S. Cao, and J. P. Hagan, "The LIN28/let-7 pathway in cancer," Frontiers in Genetics, vol. 8, p. 31, 2017.

[82] S. Wang, J. Zhan, X. Lin, Y. Wang, Y. Wang, and Y. Liu, “circRNA-0077930 from hyperglycaemia-stimulated vascular endothelial cell exosomes regulates senescence in vascular smooth muscle cells," Cell Biochemistry and Function, vol. 38, no. 8, pp. 1056-1068, 2020.

[83] S. Manzanero, T. Santro, and T. V. Arumugam, "Neuronal oxidative stress in acute ischemic stroke: sources and contribution to cell injury," Neurochemistry International, vol. 62, no. 5, pp. 712-718, 2013.

[84] T. Fumoto, M. Naraoka, T. Katagai, Y. Li, N. Shimamura, and H. Ohkuma, "The role of oxidative stress in microvascular disturbances after experimental subarachnoid hemorrhage," Translational Stroke Research, vol. 10, no. 6, pp. 684-694, 2019.

[85] S. Dong, S. Maniar, M. D. Manole, and D. Sun, "Cerebral hypoperfusion and other shared brain pathologies in ischemic stroke and Alzheimer's disease," Translational Stroke Research, vol. 9, no. 3, pp. 238-250, 2018.

[86] T. W. Kensler, N. Wakabayashi, and S. Biswal, "Cell survival responses to environmental stresses via the Keap1-Nrf2-ARE pathway," Annual Review of Pharmacology and Toxicology, vol. 47, no. 1, pp. 89-116, 2007.

[87] G. P. Sykiotis and D. Bohmann, “Stress-activated cap'n'collar transcription factors in aging and human disease," Science Signaling, vol. 3, no. 112, p. re3, 2010.

[88] J. H. Yang, R. J. Zhang, J. J. Lin et al., "The differentially expressed circular RNAs in the substantia nigra and corpus striatum of Nrf2-knockout mice," Cellular Physiology and Biochemistry, vol. 50, no. 3, pp. 936-951, 2018.

[89] C. Jahn, C. Bar, and T. Thum, "circSlc8a1, breaking a vicious circle in cardiac hypertrophy," Cardiovascular Research, vol. 115, no. 14, pp. 1946-1947, 2019.
[90] T. B. Lim, E. Aliwarga, T. D. A. Luu et al., "Targeting the highly abundant circular RNA circSlc8al in cardiomyocytes attenuates pressure overload induced hypertrophy," Cardiovascular Research, vol. 115, no. 14, pp. 1998-2007, 2019.

[91] M. Hanan, A. Simchovitz, N. Yayon et al., “A Parkinson's disease circRNAs resource reveals a link between circSLC8A1 and oxidative stress," EMBO Molecular Medicine, vol. 12, no. 9, article e11942, 2020.

[92] Q. Cheng, X. Cao, L. Xue, L. Xia, and Y. Xu, “circPRKCImiR-545/589-E2F7 axis dysregulation mediates hydrogen peroxide- induced neuronal cell injury," Biochemical and Biophysical Research Communications, vol. 514, no. 2, pp. 428-435, 2019.

[93] L. Z. Ding, J. Xu, C. Yuan, X. Teng, and Q. M. Wu, "miR-7a ameliorates spinal cord injury by inhibiting neuronal apoptosis and oxidative stress," European Review for Medical and Pharmacological Sciences, vol. 24, no. 1, pp. 11-17, 2020.

[94] H. H. Geng, R. Li, Y. M. Su et al., "The circular RNA Cdrlas promotes myocardial infarction by mediating the regulation of miR-7a on its target genes expression," PLoS One, vol. 11, no. 3, article e0151753, 2016.

[95] Y. Liu, X. Chen, J. Yao, and J. Kang, "Circular RNA ACR relieves high glucose-aroused RSC96 cell apoptosis and autophagy via declining microRNA-145-3p," Journal of Cellular Biochemistry, 2019.

[96] S. Tian, W. Zhao, D. Yang et al., "Atorvastatin inhibits miR143 expression: a protective mechanism against oxidative stress in cardiomyocytes," International Journal of Cardiology, vol. 211, pp. 115-118, 2016.

[97] S. Wang, J. Chen, W. Yu, and F. Deng, "Circular RNA DLGAP4 ameliorates cardiomyocyte apoptosis through regulating BCL2 via targeting miR-143 in myocardial ischemiareperfusion injury," International Journal of Cardiology, vol. 279, p. 147, 2019.

[98] P. Jin, L. H. Li, Y. Shi, and N. B. Hu, "Salidroside inhibits apoptosis and autophagy of cardiomyocyte by regulation of circular RNA hsa_circ_0000064 in cardiac ischemiareperfusion injury," Gene, vol. 767, article 145075, 2020.

[99] K. Wang, B. Long, F. Liu et al., "A circular RNA protects the heart from pathological hypertrophy and heart failure by targeting miR-223," European Heart Journal, vol. 37, no. 33, pp. 2602-2611, 2016.

[100] P. P. Wang, Y. J. Zhang, T. Xie, J. Sun, and X. D. Wang, "miR223 promotes cardiomyocyte apoptosis by inhibiting Foxo3a expression," European Review for Medical and Pharmacological Sciences, vol. 22, no. 18, pp. 6119-6126, 2018.

[101] M. Li, W. Ding, M. A. Tariq et al., "A circular transcript ofncxlgene mediates ischemic myocardial injury by targeting miR-133a-3p," Theranostics, vol. 8, no. 21, pp. 5855-5869, 2018.

[102] Y. Li, T. Cheng, C. Wan, and Y. Cang, “circRNA_0084043 contributes to the progression of diabetic retinopathy via sponging miR-140-3p and inducing TGFA gene expression in retinal pigment epithelial cells," Gene, vol. 747, article 144653, 2020.

[103] Y. Xu, W. Jiang, L. Zhong et al., "circ-AKT3 aggravates renal ischaemia-reperfusion injury via regulating miR-144-5p $/ \mathrm{Wnt} / \beta$-catenin pathway and oxidative stress," Journal of Cellular and Molecular Medicine, vol. 16, 2020.

[104] D. Yin, L. Liu, Z. Shi, L. Zhang, and Y. Yang, "Ropivacaine inhibits cell proliferation, migration and invasion, whereas 
induces oxidative stress and cell apoptosis by circSCAF11/miR-145-5p axis in glioma," Cancer Management and Research, vol. Volume 12, pp. 11145-11155, 2020.

[105] D. Feng, Z. Wang, Y. Zhao et al., "circ-PRKCB acts as a ceRNA to regulate p66Shc-mediated oxidative stress in intestinal ischemia/reperfusion," Theranostics, vol. 10, no. 23, pp. 10680-10696, 2020.

[106] R. Ge and G. Gao, "Anti-antioxidant impacts of circZNF609 silence in HaCaT cells through regulating miR-145," Artif Cells Nanomed Biotechnol, vol. 48, no. 1, pp. 384-392, 2020.

[107] X. Cui, Y. Dong, M. Li et al., "A circular RNA from NFIX facilitates oxidative stress-induced H9c2 cells apoptosis," In Vitro Cellular \& Developmental Biology. Animal, vol. 56, no. 9, pp. 715-722, 2020.

[108] B. Chen, Y. Li, Y. Liu, and Z. Xu, "circLRP6 regulates high glucose-induced proliferation, oxidative stress, ECM accumulation, and inflammation in mesangial cells," Journal of Cellular Physiology, vol. 234, no. 11, pp. 21249-21259, 2019.

[109] D. Chen, Z. Miao, M. Peng, H. Xing, H. Zhang, and X. Teng, "The co-expression of circRNA and mRNA in the thymuses of chickens exposed to ammonia," Ecotoxicology and Environmental Safety, vol. 176, pp. 146-152, 2019.

[110] J. Zhang, D. Li, D. Wang, K. Man, and X. Yang, "circRNA expression profiles in human dental pulp stromal cells undergoing oxidative stress," Journal of Translational Medicine, vol. 17, no. 1, p. 327, 2019. 